\title{
Five parameters for the evaluation of the soil nonlinearity during the Ms8.0 Wenchuan Earthquake using the HVSR method
}

\author{
Yefei Ren ${ }^{0}$, Ruizhi Wen* ${ }^{*}$, Xinxin Yao and Kun Ji
}

\begin{abstract}
The consideration of soil nonlinearity is important for the accurate estimation of the site response. To evaluate the soil nonlinearity during the 2008 Ms 8.0 Wenchuan Earthquake, 33 strong-motion records obtained from the main shock and 890 records from 157 aftershocks were collected for this study. The horizontal-to-vertical spectral ratio (HVSR) method was used to calculate five parameters: the ratio of predominant frequency $\left(\mathrm{RF}_{\mathrm{p}}\right)$, degree of nonlinearity (DNL), absolute degree of nonlinearity (ADNL), frequency of nonlinearity ( $\mathrm{NLL}$ ), and percentage of nonlinearity (PNL). The purpose of this study was to evaluate the soil nonlinearity level of 33 strong-motion stations and to investigate the characteristics, performance, and effective usage of these five parameters. Their correlations with the peak ground acceleration (PGA), peak ground velocity (PGV), average uppermost 30-m shear-wave velocity $\left(V_{S 30}\right)$, and maximum amplitude of HVSR $\left(A_{\max }\right)$ were investigated. The results showed that all five parameters correlate well with PGA and $P G V$. The DNL, ADNL, and PNL also show a good correlation with $A_{\max }$ which means that the degree of soil nonlinearity not only depends on the ground-motion amplitude (e.g., PGA and PGV) but also on the site condition. The fNL correlates with PGA and PGV but shows no correlation with either $A_{\max }$ or $V_{S 30}$, implying that the frequency width affected by the soil nonlinearity predominantly depends on the ground-motion amplitude rather than the site condition. At 16 of the 33 stations analyzed in this study, the site response showed evident (i.e., strong and medium) nonlinearity during the main shock of the Wenchuan Earthquake, where the ground-motion level was almost beyond the threshold of PGA $>200 \mathrm{~cm} / \mathrm{s}^{2}$ or PGV $>15 \mathrm{~cm} / \mathrm{s}$. The site response showed weak and no nonlinearity at the other 14 and 3 stations. These results also confirm that $R F_{p^{\prime}}, D N L, A D N L$, and $P N L$ are effective in identifying the soil nonlinearity behavior. The identification results vary for different parameters because each parameter has individual features. The performance of the PNL was better than that of DNL and ADNL in this case study. The thresholds of ADNL and PNL are proposed to be 2.0 and $7 \%$, respectively.
\end{abstract}

Keywords: Wenchuan Earthquake, Soil nonlinearity, Site response, HVSR method, DNL, PGA, PGV, V V

\section{Introduction}

It is well known that seismic waves can be amplified by surface soil layers that have a strong impedance contrast with deep bedrock. This is often called site response (or soil amplification) and can exacerbate earthquake damage such as in the 1985 Mexico Earthquake (Celebi et al. 1987) and 1989 Loma Prieta Earthquake in the USA

\footnotetext{
*Correspondence: ruizhi@iem.ac.cn

Key Laboratory of Earthquake Engineering and Engineering Vibration, Institute of Engineering Mechanics, China Earthquake Administration, No. 29 Xuefu Road, Harbin 150080, Heilongjiang, People's Republic of China
}

(Borcherdt and Glassmoyer 1992). It is therefore important to the earthquake engineering community to be able to accurately evaluate the site response. However, there has been a long-standing debate between geotechnical engineers and seismologists over whether site response is linearly or nonlinearly associated with ground-motion amplitude (Field et al. 1997; Beresnev and Wen 1996).

Real strong-motion records of the 1994 Northridge Earthquake in the USA were cited as direct evidence of nonlinear site response (e.g., Trifunac and Todorovska 1996; Beresnev et al. 1998; Hartzell 1998). When the ground motion exceeds a certain threshold, site response 
changes occur, reflecting a shift of the resonant frequencies toward lower values and a reduction in the associated amplification. Further evidence has been observed for many subsequent strong earthquake events such as the 1995 Kobe Earthquake in Japan (Aguirre and Irikura 1997; Pavlenko and Irikura 2002), 1999 Chi-chi Earthquake in Taiwan (Pavlenko and Loh 2005; Pavlenko and Wen 2008), 2011 Christchurch Earthquake in New Zealand (Wen et al. 2011), and 2011 Tohoku Earthquake in Japan (Bonilla et al. 2011).

Many methods were proposed in previous studies to identify the nonlinearity of the site response such as the transfer function method (Wen 1994) and horizontalto-vertical spectral ratio (HVSR) method. Because of its simplicity and ease of operation, the HVSR method has been used successfully in evaluating the nonlinear site response of several typical earthquakes, such as the 1994 Northridge Earthquake (Dimitriu 2002) and 2010 Darfield Earthquake sequence in New Zealand (Wen et al. 2011), and some observational networks such as the Kyoshin network (K-net) and Kiban Kyoshin network (KiK-net; Régnier et al. 2013, 2016).

The HVSR method has also been used to evaluate the nonlinearity of the site response of the 2008 Ms8.0 Wenchuan Earthquake. For example, using the HVSR method together with the short-term Fourier transform, $\mathrm{Xu}$ (2010) identified clear soil nonlinearity for sites that recorded peak ground acceleration (PGA) $>200 \mathrm{~cm} / \mathrm{s}^{2}$. It was found that these sites were located in areas where soil liquefaction was observed. Rong et al. (2016) used the HVSR method to investigate the nonlinear site response at 21 strong-motion stations. They found that the predominant frequency decreased with increasing groundmotion level, but they did not observe a decrease in the amplitude of the soil amplification because of limited data. However, these studies did not systematically investigate the features of some parameters that could be used to quantitatively evaluate the level of soil nonlinearity such as the degree of nonlinearity (DNL). This parameter was defined by Noguchi and Sasatani (2008) and has been used in many case studies such as for the identification of the soil nonlinearity of strong-motion sites at the ocean bottom (Dhakal et al. 2017).

The objective of this study was to evaluate the level of soil nonlinearity of 33 strong-motion stations using five parameters and to identify the PGA and peak ground velocity (PGV) thresholds beyond which the site response evidently behaved nonlinearly during the Wenchuan main shock. These parameters include DNL, frequency of nonlinearity (fNL), and percentage of nonlinearity (PNL), defined by Régnier et al. (2013), and the ratio of the predominant frequency $\left(\mathrm{RF}_{\mathrm{p}}\right)$ and absolute degree of nonlinearity (ADNL), as defined in this study.
The second objective of this study was to investigate the characteristics, performance, and effective usage of these five parameters to evaluate the nonlinear behavior of the site response. The correlations between these five parameters and PGA, PGV, average uppermost 30-m shear-wave velocity $\left(V_{\mathrm{S} 30}\right)$, and maximum amplitude of HVSR $\left(A_{\max }\right)$ were investigated to analyze the effect of the site condition and ground-motion amplitude on the degree and frequency width of the soil nonlinearity.

\section{Dataset and data processing}

More than 400 strong-motion records were collected during the main shock of the 2008 Ms8.0 Wenchuan Earthquake (Li et al. 2008), and more than 2000 records were acquired from 383 aftershocks (Li 2009). Several large PGAs were recorded, for example, $957.3 \mathrm{~cm} / \mathrm{s}^{2}$ at station $51 \mathrm{WCW},-824.6 \mathrm{~cm} / \mathrm{s}^{2}$ at station $51 \mathrm{MZQ}$, and $-585.7 \mathrm{~cm} / \mathrm{s}^{2}$ at station 51SFB. A large ground-motion amplitude might trigger the change of the site response from the linear to nonlinear stage.

The HVSRs, representative of soil amplification, were calculated using the strong-motion records obtained from the main shock (i.e., strong motion) and aftershocks (i.e., weak motion), separately. Here, strong motion is defined as record with PGA $>100 \mathrm{~cm} / \mathrm{s}^{2}$, observed in any one of the components during the main shock of the Wenchuan Earthquake. Consequently, 33 strongmotion stations were selected; their locations are shown in Fig. 1. To provide reliable estimates of PGA and PGV, a baseline correction was made to these 33 records using the method proposed by Boore (2010). The station code, PGA, PGV, and $V_{\mathrm{S} 30}$ (proxy for the site condition) of each station are listed in Table 1.

For weak motions, the records obtained from hundreds of aftershocks were selected based on the following criteria: (1) The geometric mean of PGA of two horizontal components should be $>2$ and $<100 \mathrm{~cm} / \mathrm{s}^{2}$. The lower boundary is assigned to avoid noise contamination and the upper boundary value is set to remove data potentially affected by soil nonlinearity. Many studies reported that the threshold of PGA causing nonlinear site response is $>100 \mathrm{~cm} / \mathrm{s}^{2}$ (e.g., Régnier et al. 2016; Dhakal, et al. 2017); (2) to ensure a relatively low level of scattering of the HVSR results, each station should provide at least three records that match criterion (1).

Overall, 890 records from 157 aftershocks were available for this study. Figure 1 shows the epicenters of these aftershocks and the locations of the corresponding triggered stations. The magnitude-distance and magnitudePGA distributions of these records are shown in Fig. 2, clearly illustrating that the records have uniform distribution within a range $<200 \mathrm{~km}$. 


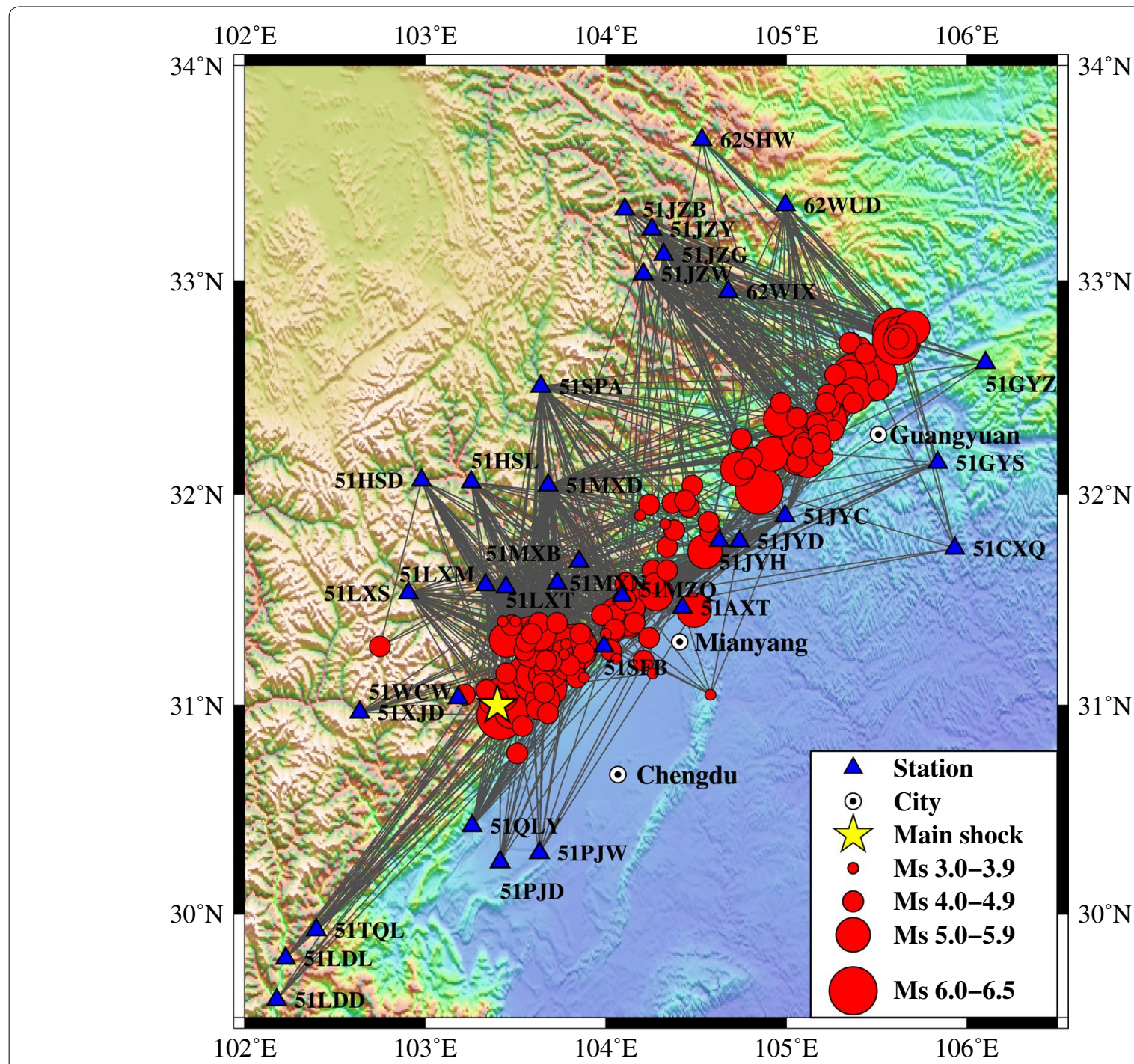

GMJ 2017 May 30 14:31:03

Fig. 1 Locations of strong-motion stations and earthquake epicenters selected in this study. The gray line is the path between the source and site. The source parameters were obtained from a catalogue database of the China Earthquake Networks Center (http://www.csndmc.ac.cn/)

A Butterworth filter with a bandwidth of $0.3-25.0 \mathrm{~Hz}$ was applied to each record. The $\mathrm{S}$-wave portion was then detected following the method of Ren et al. (2013). To remove truncation errors, a cosine-type tapered window was used. The Fourier amplitude spectrum (FAS) for each component was calculated. A 0.5-Hz-width Parzen window was then used to smooth the spectrum. Finally, the resultant horizontal FAS $H(f)$ was determined based on the geometric mean using Eq. (1), where $H_{1}(f)$ and $H_{2}(f)$ represent the FAS of two orthogonal horizontal components:

$$
\ln H(f)=\frac{\ln H_{1}(f)+\ln H_{2}(f)}{2}
$$

\section{Site response calculated with the HVSR method}

The site responses of the 33 stations in the frequency range of $0.5-20.0 \mathrm{~Hz}$ were calculated individually using the HVSR method for strong and weak motions, as shown in Figs. 3 and 4. Multiple HVSR curves produced a geometric mean, which was used to determine the site response under weak motions, while only one curve was available for strong motions. It clearly shows that site response under strong motion is considerably smaller than that under weak motion in the high-frequency band for stations 51GYS, 51GYZ, 51JYC, 51JYD, 51JYH, $51 \mathrm{SFB}$, and $51 \mathrm{WCW}$, and the predominant frequency $\left(F_{\mathrm{p}}\right)$ significantly shifts from high to low frequency. This 


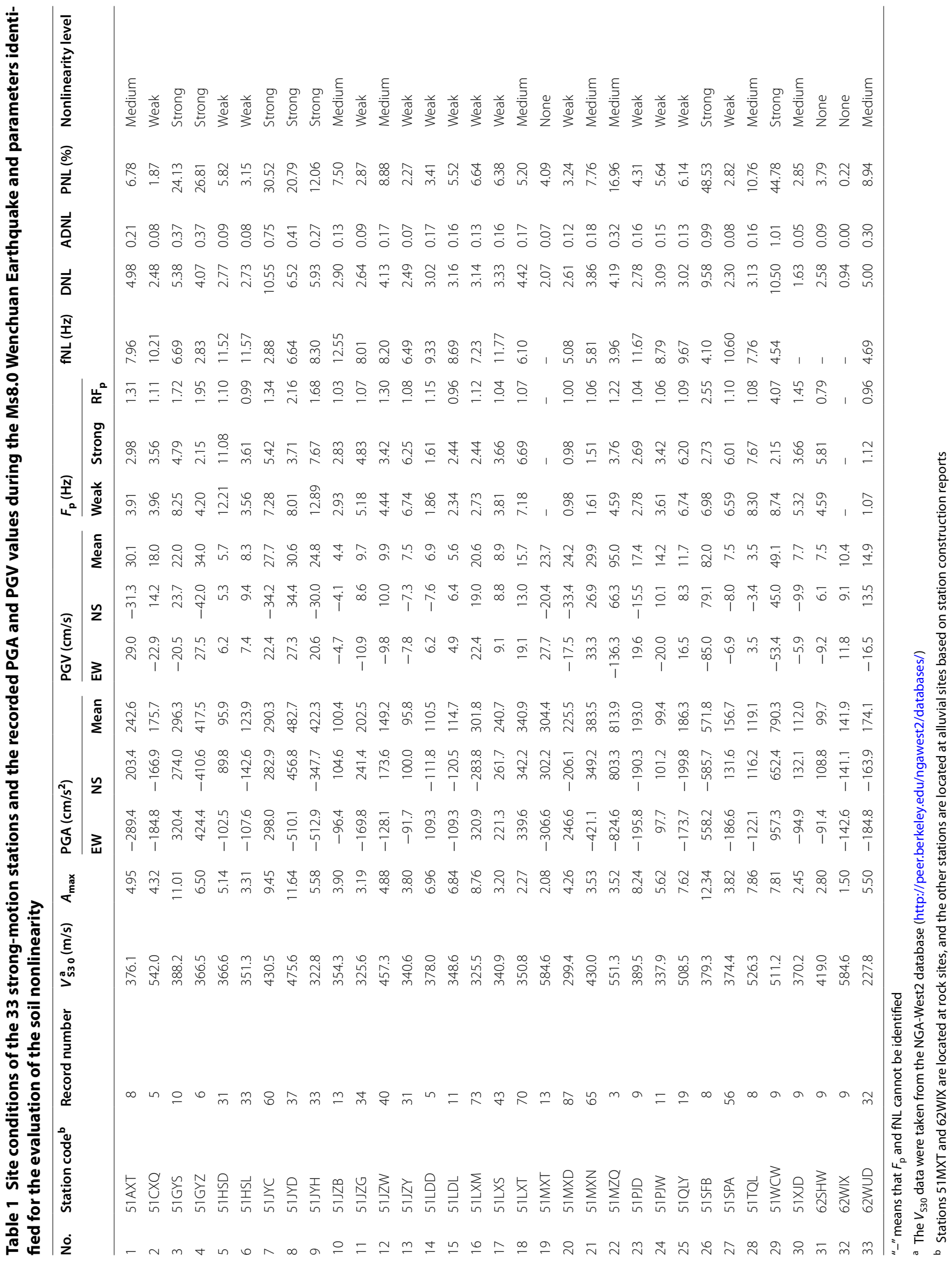




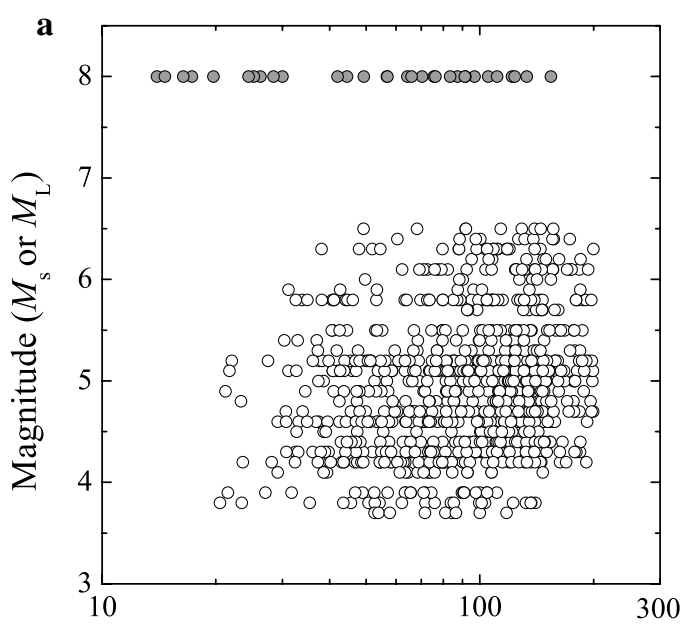

Hypocenter or Rupture Distance $(\mathrm{km})$

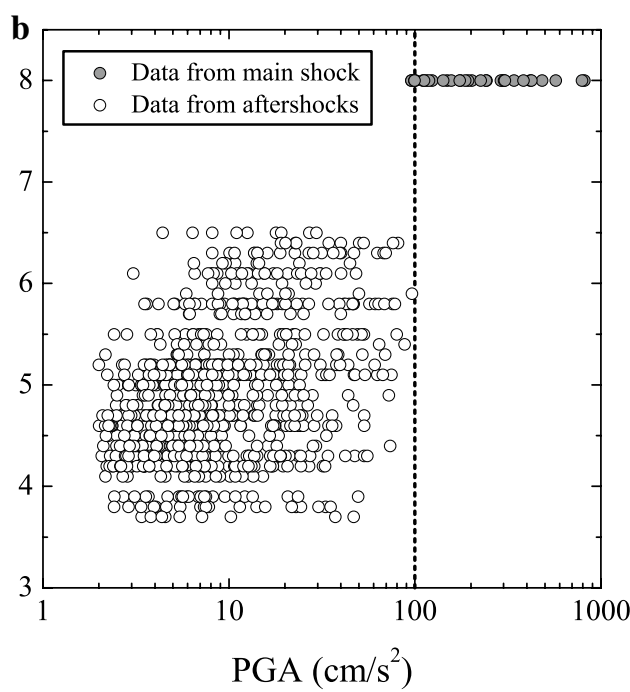

Fig. 2 Magnitude versus $\mathbf{a}$ hypocenter or rupture distance and $\mathbf{b}$ PGA of the strong-motion records used in this study. The hypocenter distance is used for aftershocks, and the rupture distance (shortest distance between the station and rupture surface) is used for the main shock, which is calculated according to the fault slip model provided by the USGS. The vertical dashed line is the boundary between strong and weak motions defined in this study

implies that strong soil nonlinearity occurred during the main shock at these stations. Although less evident, a similar phenomenon can be observed at stations 51AXT, 51JZW, 51LXS, 51QLY, 51TQL, and 62WUD, implying medium or weak nonlinearity.

The values of PGA and PGV, recorded during the main shock, and $V_{\mathrm{S} 30}$ for each station are also presented in Figs. 3 and 4. It can be observed that the sites at which a small PGA or PGV was recorded, such as 51HSD, 51HSL, 51LDD, 51LDL, 51XJD, and 62SHW, show weak evidence of soil nonlinearity. Furthermore, there is absolutely no evidence of soil nonlinearity for sites without covering soil such as 51MXT and 62WIX. According to station construction reports, only 51MXT and 62WIX are located at rock sites. The other stations are located at alluvial sites. Therefore, the correlations of the nonlinearity with PGA, PGV, $V_{\mathrm{S} 30}$, and $A_{\max }$ should be analyzed in detail considering the purpose of this study.

\section{Definition of the five parameters used to evaluate the soil nonlinearity}

To investigate the effects of the nonlinear behavior of soils on the site response, Régnier et al. (2013) proposed using two parameters per event and four parameters per site based on various earthquake records from the KiKnet database in Japan. The fNL and PNL were proposed by their study, the DNL was proposed by Noguchi and Sasatani (2008), and $\mathrm{RF}_{\mathrm{p}}$ and ADNL are proposed in this study.

The parameter DNL is defined as follows:

$$
\mathrm{DNL}=\sum_{i=N_{1}}^{N_{2}}\left|\log \left[\frac{R_{\text {strong }}(i)}{R_{\text {weak }}(i)}\right]\right|\left(f_{i+1}-f_{i}\right),
$$

where $R_{\text {strong }}(i)$ and $R_{\text {weak }}(i)$ represent the HVSR values at the frequency $f_{i}$ for $\mathrm{S}$-waves during strong (PGA $>100 \mathrm{~cm} / \mathrm{s}^{2}$ in this study) and weak motions (PGA $<100 \mathrm{~cm} / \mathrm{s}^{2}$ in this study), respectively, and $N_{1}$ and $\mathrm{N}_{2}$ represent the beginning and ending frequencies, usually set as 0.5 and $20.0 \mathrm{~Hz}$. Figure $5 \mathrm{a}$ shows an illustration of the calculation of DNL, which actually represents the non-overlapping parts of both areas enclosing the HVSRs for S-waves during strong and weak motions and the horizontal ordinate of the frequency.

It should be noted that the average HVSR for S-waves during weak motion is used when calculating the value of DNL; however, its standard deviation is not included. To consider the effect of the standard deviation, we propose an improved parameter called the absolute degree of nonlinearity (ADNL), which is defined as follows:

$$
\mathrm{ADNL}=\sum_{i=N_{1}}^{N_{2}} \Delta(i) \cdot\left[\log \left(f_{i+1}\right)-\log \left(f_{i}\right)\right]
$$

$$
\Delta(i)= \begin{cases}\log \left(R_{\text {strong }}(i)\right)-\log \left(R_{\text {weak }}^{+}(i)\right) & R_{\text {strong }}(i) \geq R_{\text {weak }}^{+}(i) \\ \log \left(R_{\text {weak }}^{-}(i)\right)-\log \left(R_{\text {strong }}(i)\right) & R_{\text {strong }}(i) \leq R_{\text {weak }}^{-}(i) \\ 0 & \text { others }\end{cases}
$$



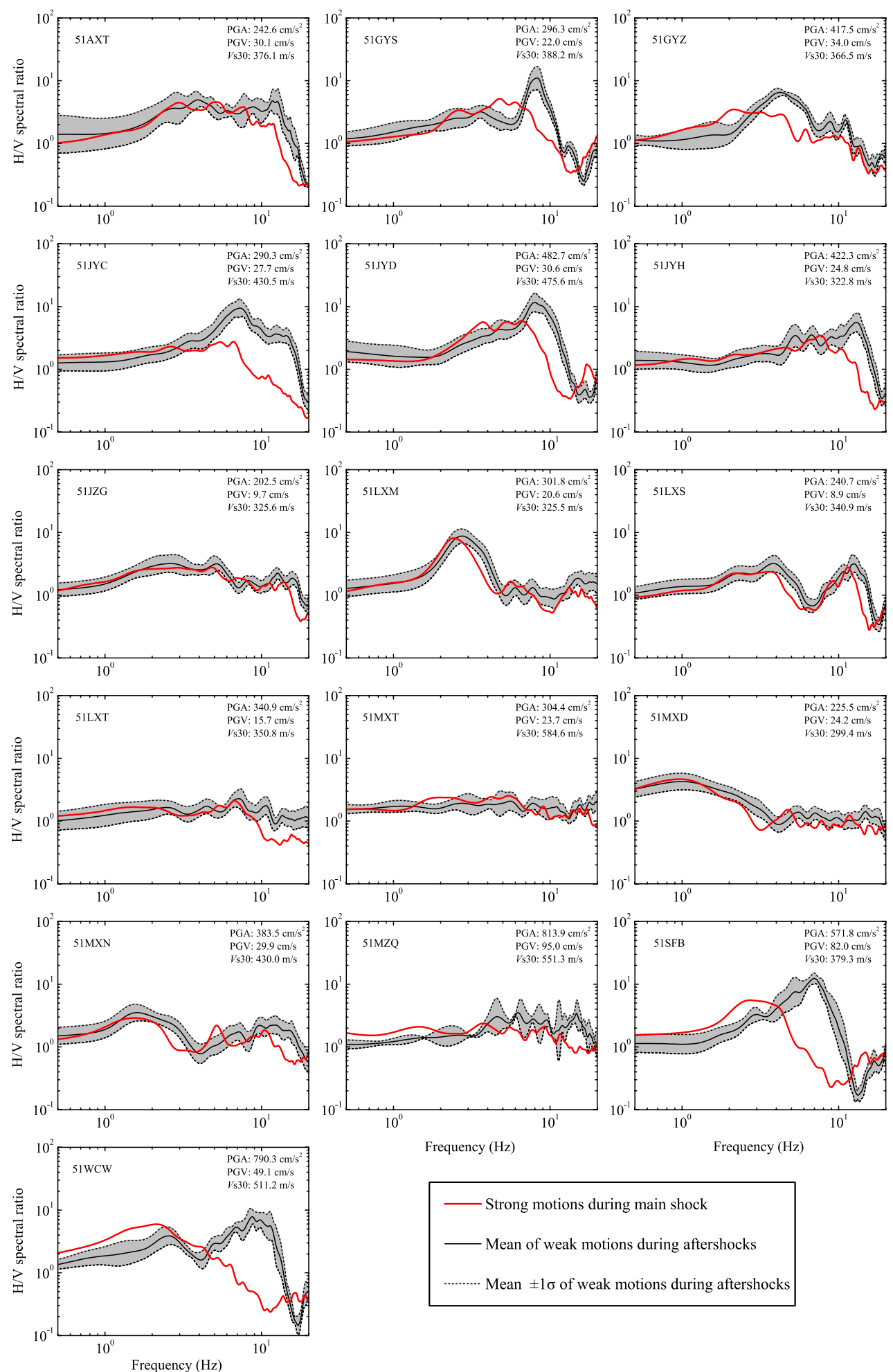

Fig. 3 Site responses calculated using the HVSR method for 16 strong-motion stations with PGA $>200 \mathrm{~cm} / \mathrm{s}^{2}$ under strong and weak motion during the $2008 \mathrm{Ms} 8.0$ Wenchuan Earthquake sequence. The $V_{S 30}, P G A$, and PGV values recorded during the main shock for each station are presented. The shaded area indicates the range of the mean plus-minus one standard deviation 

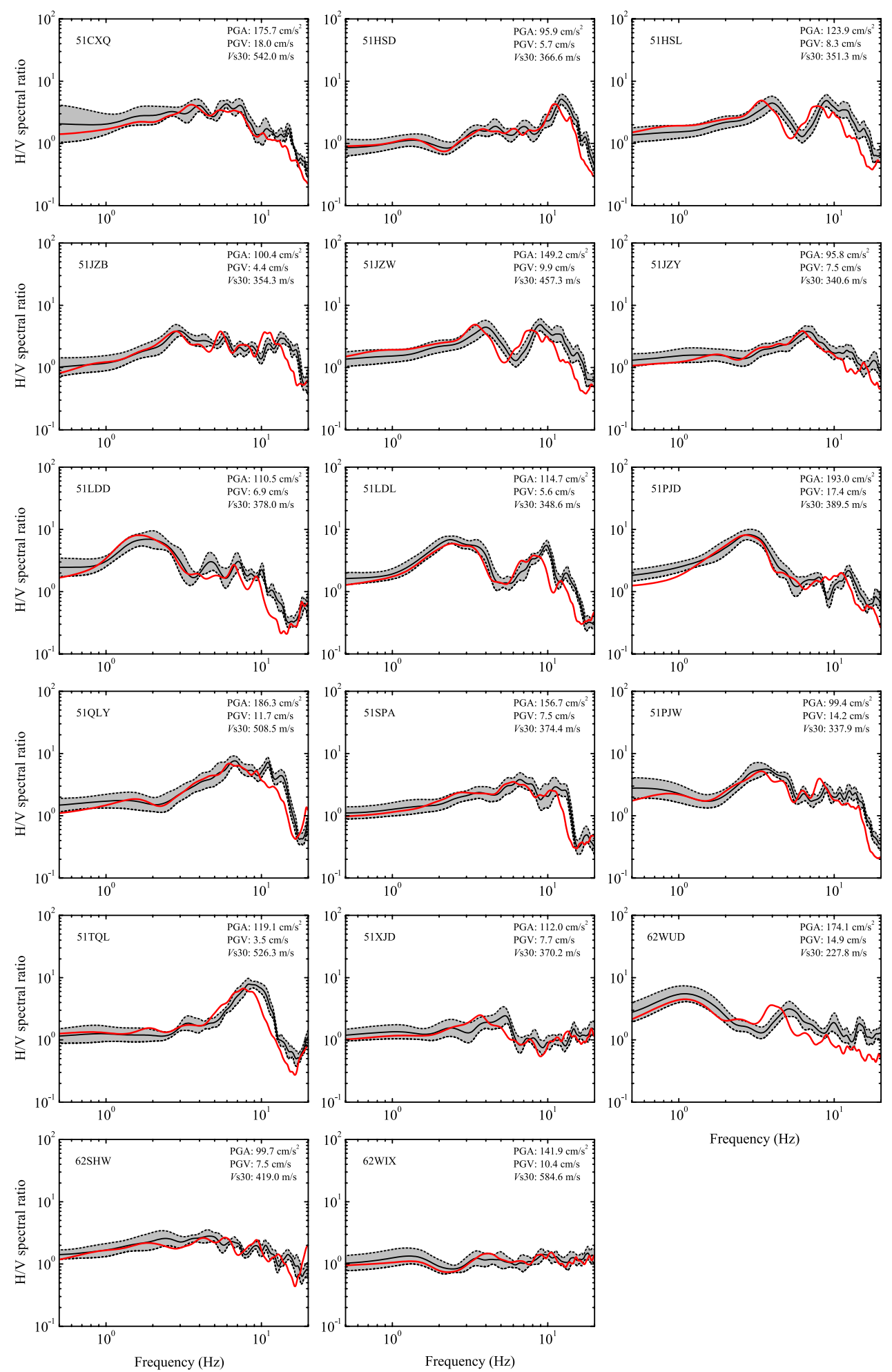

Fig. 4 Same as Fig. 3 but for 17 stations with PGA $<200 \mathrm{~cm} / \mathrm{s}^{2}$ 

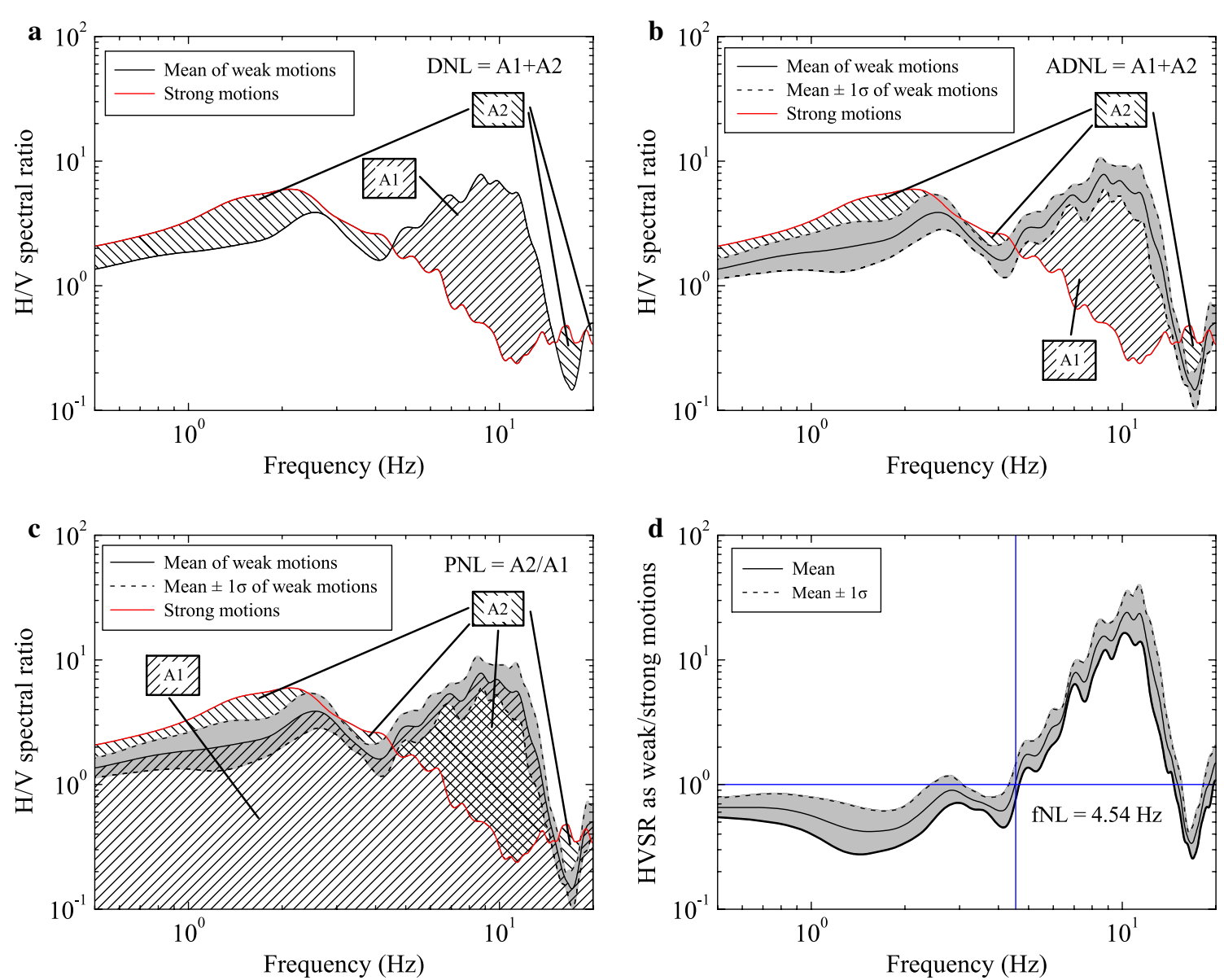

Fig. 5 Illustration of the calculation of $\mathbf{a} \mathrm{DNL}, \mathbf{b} A D N L, \mathbf{c} P N L$, and $\mathbf{d}$ fNL based on the example of site $51 \mathrm{WCW}$

where $R_{\text {weak }}^{+}(i)$ and $R_{\text {weak }}^{-}(i)$ represent the values of the average HVSR plus-minus one standard deviation at the frequency $f_{i}$ for $\mathrm{S}$-waves during weak motion. To balance the contributions from high and low frequencies, the frequency interval was calculated on a logarithmic scale. An illustration of the calculation of ADNL is shown in Fig. $5 \mathrm{~b}$ based on the example of site $51 \mathrm{WCW}$.

The definition of PNL proposed by Régnier et al. (2013) is as follows:

$$
\begin{aligned}
& \mathrm{PNL}=A_{2} / A_{1} \\
& A_{1}=\sum_{i=N_{1}}^{N_{2}} R_{\text {weak }}(i) \cdot\left[\log \left(f_{i+1}\right)-\log \left(f_{i}\right)\right] \\
& A_{2}=\sum_{i=N_{1}}^{N_{2}} \delta(i) \cdot\left[\log \left(f_{i+1}\right)-\log \left(f_{i}\right)\right]
\end{aligned}
$$

$\delta(i)= \begin{cases}R_{\text {strong }}(i)-R_{\text {weak }}^{+}(i) & R_{\text {strong }}(i) \geq R_{\text {weak }}^{+}(i) \\ R_{\text {weak }}^{-}(i)-R_{\text {strong }}(i) & R_{\text {strong }}(i) \leq R_{\text {weak }}^{-}(i) \\ 0 & \text { others }\end{cases}$

An illustration of how to calculate PNL is shown in Fig. $5 \mathrm{c}$ for the example of station $51 \mathrm{WCW}$, where $A_{1}$ is the area enclosing the HVSR curve derived from weak motion and the horizontal ordinate of the frequency, $A_{2}$ is the area difference between the two HVSR curves derived from weak and strong motions, and PNL represents the percentage of the relative change from linear to nonlinear site response.

Based on the definitions of DNL, ADNL, and PNL, all parameters characterize one feature of soil nonlinearity, that is, the reduction in the site response amplitude at high frequency. In fact, the frequency dependence of the effects of soil nonlinearity has been underlined in previous studies (e.g., Wen et al. 1994; Delépine et al. 2009). Régnier et al. (2013) defined the parameter fNL to reveal another feature of soil nonlinearity, that is, 
the shift of the predominant frequency toward lower values.

Figure $5 \mathrm{~d}$ shows an illustration of the calculation of $\mathrm{fNL}$ based on the example of site 51WCW. Taking a ratio of the HVSR of the S-wave during weak motion over that during strong motion, amplification of the nonlinear response can be observed below a given frequency compared with the linear case and deamplification takes place above this given frequency. This critical frequency is determined as the value of fNL. Notably, if fNL is smaller, it is probable that the frequency band affected by soil nonlinearity will be wider.

To clearly reveal the shift degree of the predominant frequency from linear to nonlinear site response, we defined a new parameter, $\mathrm{RF}_{\mathrm{p}}$, which is the ratio of the predominant frequency $\left(F_{\mathrm{p}}\right)$ under weak and strong motions:

$$
\mathrm{RF}_{\mathrm{p}}=F_{\mathrm{p}, \text { weak }} / F_{\mathrm{p}, \text { strong, }}
$$

where $F_{\mathrm{p} \text {, weak }}$ and $F_{\mathrm{p} \text {, strong }}$ represent the predominant frequencies identified by HVSR curves calculated using weak and strong motions, respectively.

\section{Evidence of soil nonlinearity during the Wenchuan main shock}

The values of $\mathrm{RF}_{\mathrm{p}}$, DNL, ADNL, $\mathrm{NL}$, and PNL for each site were calculated (Table 1). Based on these parameters, sites showing nonlinear behavior were identified; the correlations between these parameters and PGA, PGV, $V_{\mathrm{S} 30}$, and $A_{\max }$ were investigated.

\section{Parameter $\mathrm{RF}_{\mathrm{p}}$}

Figure 6 shows the parameter $\mathrm{RF}_{\mathrm{p}}$ plotted versus the mean PGA and PGV of the two horizontal components. Note that for PGA $>200 \mathrm{~cm} / \mathrm{s}^{2}$ or PGV $>20 \mathrm{~cm} / \mathrm{s}, F_{\mathrm{p}}$ under strong motion becomes much smaller than under weak motion at most sites, representative of soil nonlinearity. This phenomenon was identified at sites $51 \mathrm{MZQ}, 51 \mathrm{WCW}$, 51SFB, 51JYD, 51GYZ, 51JYH, 51GYS, 51JYC, 51AXT, 51JZW, and 51XJD. The threshold of PGA used here is the same as that reported by $\mathrm{Xu}$ (2010) who evaluated the soil nonlinearity during the Wenchuan Earthquake using the time-frequency analysis technique. Considering the shaded area of Fig. 6, it is evident that the level of nonlinearity increases as the PGA value increases, implying strong dependence on the ground-motion amplitude.

It should be noted that the site response at station 51MZQ does not indicate soil nonlinearity behavior as strongly as station $51 \mathrm{WCW}$, even though the PGA is $>800 \mathrm{~cm} / \mathrm{s}^{2}$ and the value of PGV is close to $100 \mathrm{~cm} / \mathrm{s}$. The surface geology at station 51MZQ shows only a 1.5 -m-thick overburden above the soft bedrock (shear velocity $\sim 400 \mathrm{~m} / \mathrm{s}$ ), indicative of low possibility of soil nonlinearity (Ren et al. 2013). This indicates that the soil nonlinearity might be correlated with the site condition.

We compared the values of $\mathrm{RF}_{\mathrm{p}}$ of this study with those provided by Ren et al. (2013) who used the generalized inversion technique. As shown in Fig. 7, the values produced by the two methods are similar for most stations, implying that our results are reliable and that our approach for the identification of the soil nonlinearity is acceptable.

\section{Parameter DNL}

The linear relationships between DNL and PGA and PGV were regressed, respectively, as shown in Fig. 8a:

$$
\begin{array}{ll}
\mathrm{DNL}=5.550 \cdot \log (\mathrm{PGA})-8.916 & (R=0.65) \\
\mathrm{DNL}=4.115 \cdot \log (\mathrm{PGV})-0.840 \quad(R=0.61)
\end{array}
$$
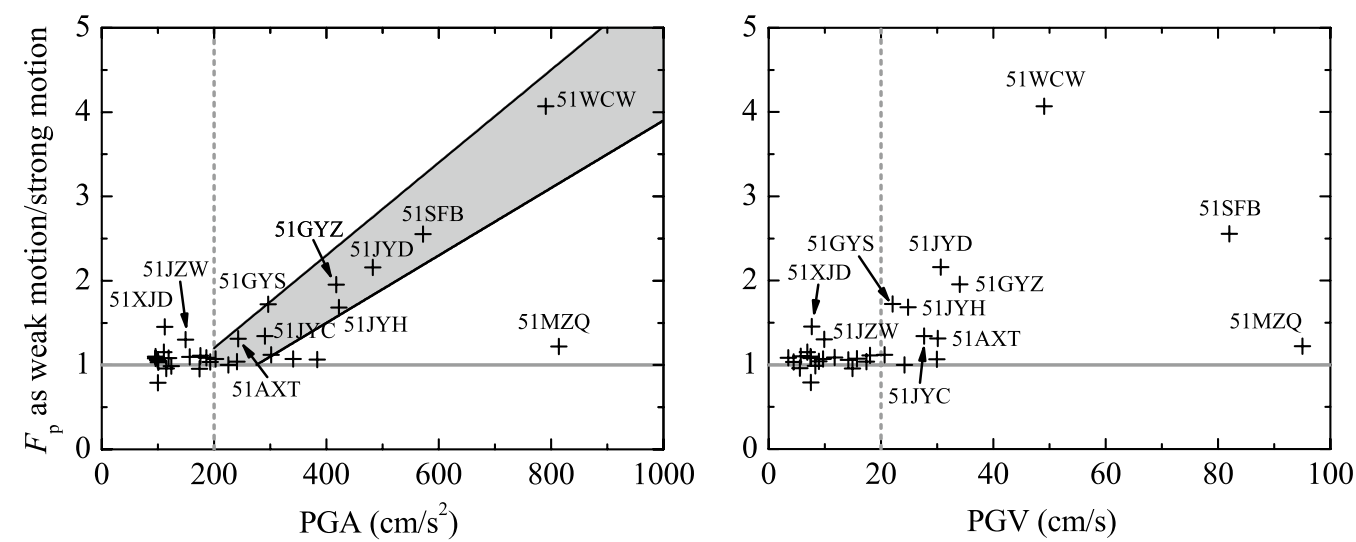

Fig. 6 Predominant frequency $F_{p}$ as weak motion over strong motion versus $\mathbf{a}$ PGA and $\mathbf{b}$ PGV. The dashed lines indicate the threshold values of $P G A$ and $P G V$ beyond which the site response is nonlinear. The shaded area shows the variation of the level of nonlinearity with PGA 


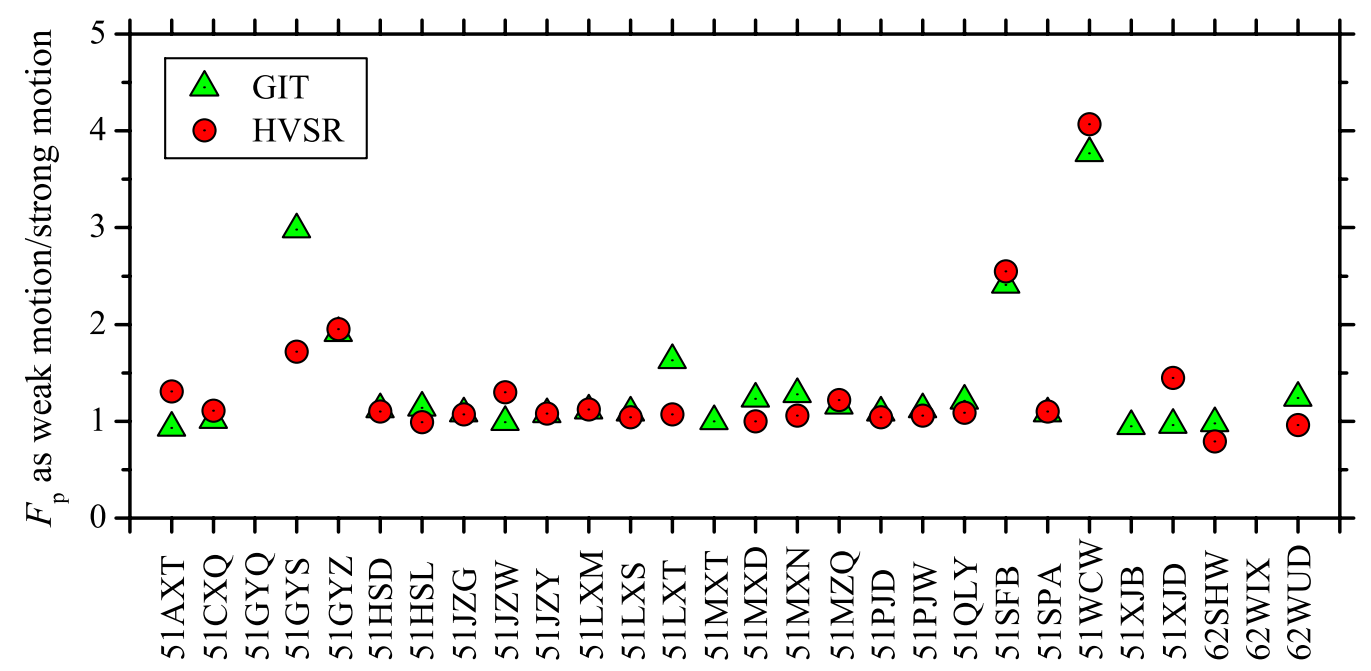

Site of strong-motion station

Fig. 7 Comparison of $F_{p}$ as weak motion over strong motion based on the HVSR method in this study and the generalized inversion technique of Ren et al. (2013)

Because the values of DNL were calculated using the logarithmic expression of HVSR (see Eq. 2), the regressions used DNL values on a linear scale but PGA and PGV values on a logarithmic scale. In the following regressions, linear scale was also used for $\mathrm{ADNL}$ and PNL but logarithmic scale used for PGA, PGV, and $A_{\max }$. It shows that DNL positively correlates with PGA and PGV. The regression correlation coefficients of 0.65 and 0.61 indicate a moderate correlation. This is in accordance with conclusions reached in previous studies (e.g., Wen et al. 2011; Dhakal et al. 2017).

Noguchi and Sasatani (2011) suggested a DNL value of 4.0 to identify the nonlinear site response, which has been used in several previous studies such as Dhakal et al. (2017). Based on this DNL value, it could be assessed that 12 stations exhibited soil nonlinearity (Fig. 8a): 51MZQ, 51WCW, 51SFB, 51JYD, 51JYC, 51JYH, 51GYZ, 51GYS, 51LXT, 51AXT, 51JZW, and 62WUD. Most of these can also be identified using parameter $\mathrm{RF}_{\mathrm{p}}$.

To understand the dependence of soil nonlinearity on the site condition, we investigated the correlation between $\mathrm{DNL}$ and $V_{\mathrm{S} 30}$, as shown in Fig. 9a. The values of $V_{\mathrm{S} 30}$ were derived from the NGA-West2 database (Ancheta et al. 2013). To eliminate the effect of the ground-motion amplitude as much as possible, the data were separated into three groups based on different PGA levels: PGA $<200 \mathrm{~cm} / \mathrm{s}^{2}$, $200<\mathrm{PGA}<400 \mathrm{~cm} / \mathrm{s}^{2}$, and PGA $>400 \mathrm{~cm} / \mathrm{s}^{2}$. However, whichever group was used, a poor relationship between DNL and $V_{\mathrm{S} 30}$ was observed. The values of $V_{\mathrm{S} 30}$ in the NGA-West2 database for sites in southwest China were estimated using an extrapolation procedure based on soil profiles at depths shallower than $30 \mathrm{~m}$. It was confirmed that the values were overestimated for sites with low shearwave velocity and underestimated for sites with high shearwave velocity (Ancheta et al. 2013). This estimation bias might be the cause of unreliable results of the analysis of the correlation between DNL and $V_{\mathrm{S} 30}$.

As an alternative to $V_{\mathrm{S} 30}$, we used $A_{\max }$ calculated using weak motion because $A_{\max }$ can be considered as a proxy for the site condition. It is known that greater soil amplification can be generated at sites with a stronger impedance contrast between the surface soil layers and bedrock. Generally, large values of $A_{\max }$ are achieved at sites with soft soil and small values of $A_{\max }$ are obtained at sites with rigid soil or outcrops. The values of DNL versus $A_{\max }$ are presented in Fig. 9b. An empirical relationship between them was regressed by linear fitting for each group of data. The results are as follows: when PGA $>400 \mathrm{~cm} / \mathrm{s}^{2}$

$$
\mathrm{DNL}=8.337 \cdot \log \left(A_{\max }\right)-0.364 \quad(R=0.63)
$$

when $200<\mathrm{PGA}<400 \mathrm{~cm} / \mathrm{s}^{2}$

$$
\mathrm{DNL}=5.554 \cdot \log \left(A_{\max }\right)+0.679 \quad(R=0.59)
$$

when $\mathrm{PGA}<200 \mathrm{~cm} / \mathrm{s}^{2}$

$$
\mathrm{DNL}=2.803 \cdot \log \left(A_{\max }\right)+0.988 \quad(R=0.64)
$$

The respective correlation coefficient $(R)$ of $0.63,0.59$, and 0.64 for each regression indicates a moderate correlation between DNL and $A_{\max }$. This implies that the degree of soil nonlinearity significantly depends on the site condition. 

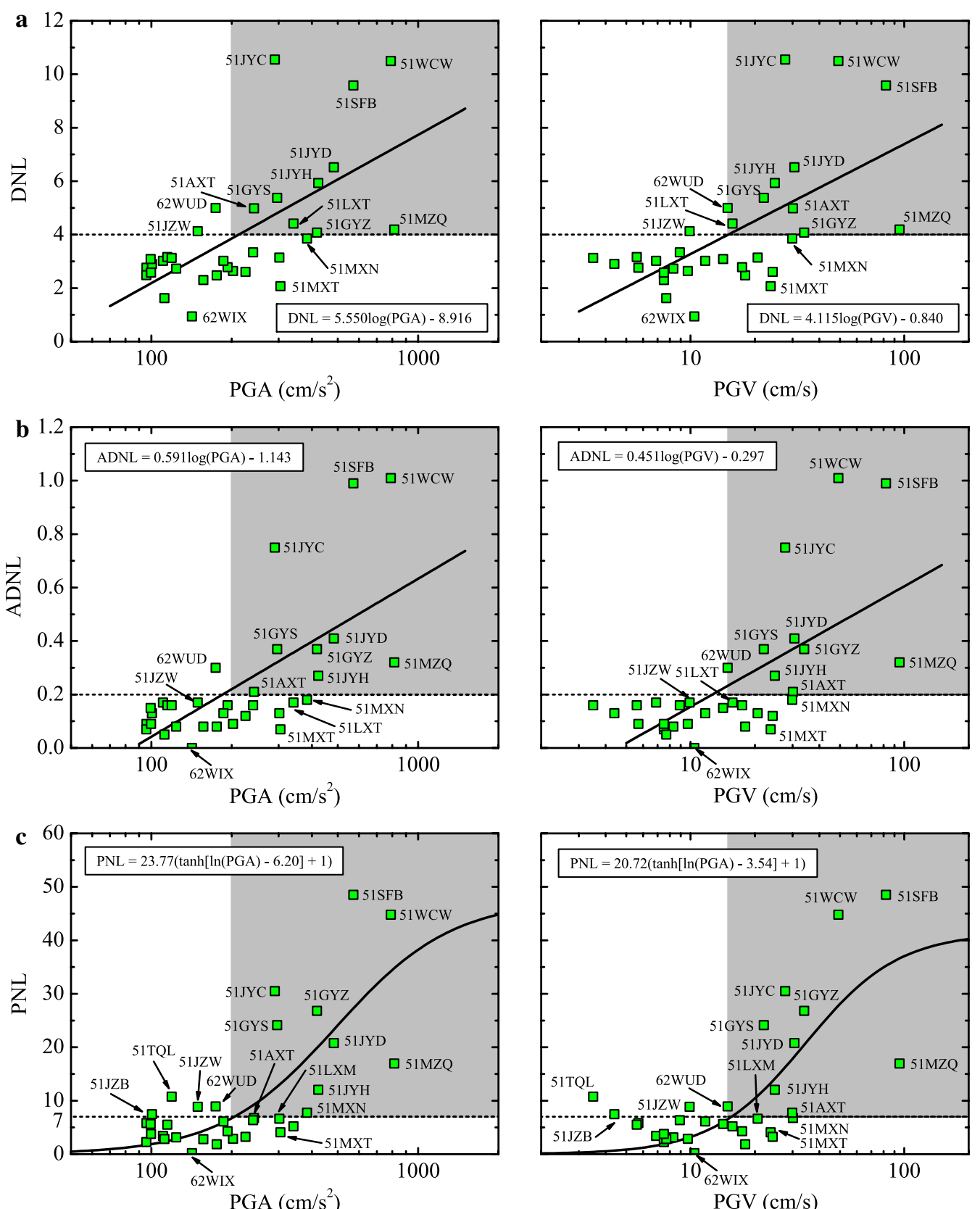

Fig. 8 Values of a DNL, b ADNL, and $\mathbf{c}$ PNL for the 33 stations versus the recorded PGAs and PGVs during the Wenchuan Earthquake including empirical relationship fitting. The dashed lines indicate the threshold of DNL, ADNL, and PNL proposed in this study (i.e., 4.0, 0.2, and 7\%) beyond which the site response exhibits evident nonlinear behavior. The shaded areas indicate the regions covering the values of DNL (or ADNL, PNL) and PGA (or PGV) beyond their thresholds. The PGA and PGV thresholds are proposed to be $200 \mathrm{~cm} / \mathrm{s}^{2}$ and $15 \mathrm{~cm} / \mathrm{s}$, respectively

Parameter ADNL

The empirical linear relationships between ADNL and PGA and PGV were also regressed (Fig. 8b):

$$
\begin{aligned}
& \mathrm{ADNL}=0.591 \cdot \log (\mathrm{PGA})-1.143 \quad(R=0.66) \\
& \mathrm{ADNL}=0.451 \cdot \log (\mathrm{PGV})-0.297 \quad(R=0.65)
\end{aligned}
$$

The $R$ value is 0.66 and 0.65 , respectively, indicating moderate correlations, similar to the relationships between DNL and PGA and PGV. The empirical relationships between ADNL and $A_{\max }$ were also regressed (Fig. 9c), implying moderate correlation. 

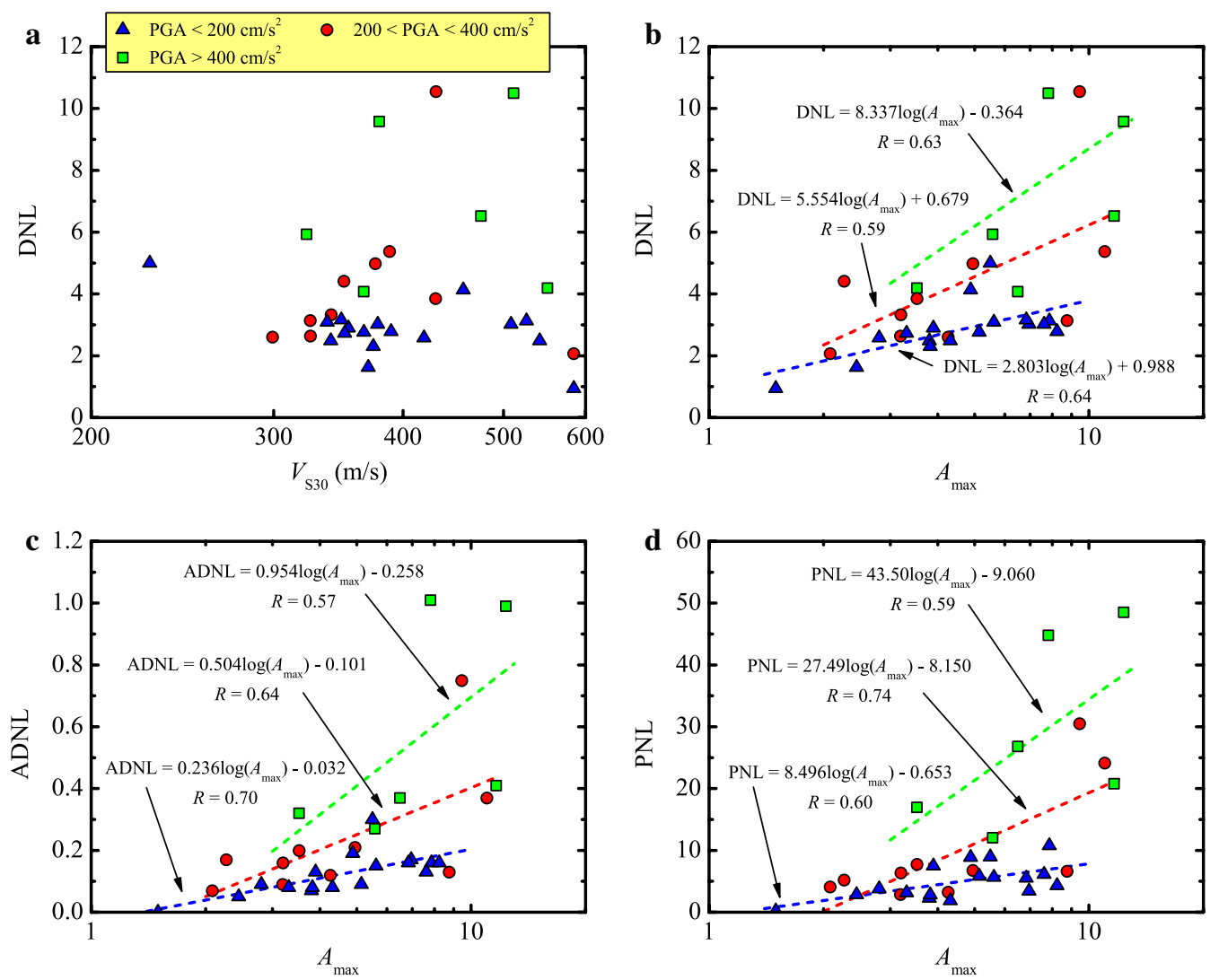

Fig. 9 a $D N L$ versus $V_{530}, \mathbf{b} D N L$ versus $A_{\max } \mathbf{c} A D N L$ versus $A_{\max }$ and $\mathbf{d}$ PNL versus $A_{\max }$. The data were separated into three groups based on different PGA levels: $P G A<200 \mathrm{~cm} / \mathrm{s}^{2}, 200<P G A<400 \mathrm{~cm} / \mathrm{s}^{2}$, and PGA $>400 \mathrm{~cm} / \mathrm{s}^{2}$. The relationships between DNL and $A_{\max }$ ADNL and $A_{\max }$ and PNL and $A_{\max }$ for each group of data are presented based on linear fitting on a logarithmic scale. The correlation coefficient $R$ is also given for each regression

\section{Parameter fNL}

Table 1 shows the value of $\mathrm{NNL}$ for each site. Note that fNL could not be identified at four sites (51MXT, 51XJD, $62 \mathrm{SHW}$, and 62WIX) because of the small difference of the HVSR during strong and weak motions, implying the absence of soil nonlinearity.

The dependence of $\mathrm{NL}$ on PGA and PGV was investigated, as shown in Fig. 10a, b. To eliminate the effect of the site condition as much as possible, the data were separated into two groups based on different $A_{\max }$ levels: $A_{\max }<5.0$ and $A_{\max }>5.0$. The reason behind using $A_{\max }$ rather than $V_{\mathrm{S} 30}$ was already explained for the analysis of DNL. The empirical relationships between $\mathrm{fNL}$ and PGA and PGV were regressed for each group of data:

when $A_{\max }>5.0$

$$
\begin{array}{lr}
\log (\mathrm{fNL})=-0.405 \cdot \log (\mathrm{PGA})+1.781 & (R=0.61) \\
\log (\mathrm{fNL})=-0.336 \cdot \log (\mathrm{PGV})+1.234 & (R=0.64)
\end{array}
$$

when $A_{\max }<5.0$

$$
\begin{array}{rr}
\log (\mathrm{fNL})=-0.423 \cdot \log (\mathrm{PGA})+1.877 & (R=0.69) \\
\log (\mathrm{fNL})=-0.342 \cdot \log (\mathrm{PGV})+1.291 & (R=0.78)
\end{array}
$$

The respective value of $R$ is larger than 0.6 for each regression, showing moderate correlations between $\mathrm{fNL}$ and PGA and PGV. The value of $\mathrm{NNL}$ decreases as PGA increases, consistent with the above-mentioned observation that $\mathrm{RF}_{\mathrm{p}}$ depends on PGA. The value of $\mathrm{RF}_{\mathrm{p}}$ increases as PGA increases, which means that the predominant frequency shifts toward the low-frequency band, which could cause a wider frequency band affected by soil nonlinearity and consequently a lower $\mathrm{fNL}$ value.

The dependences of $\mathrm{fNL}$ on $V_{\mathrm{S} 30}$ and $A_{\max }$ were investigated, as shown in Fig. 10c, d, respectively. The data were also separated into three groups based on different PGA levels. The figures show that there is no correlation 

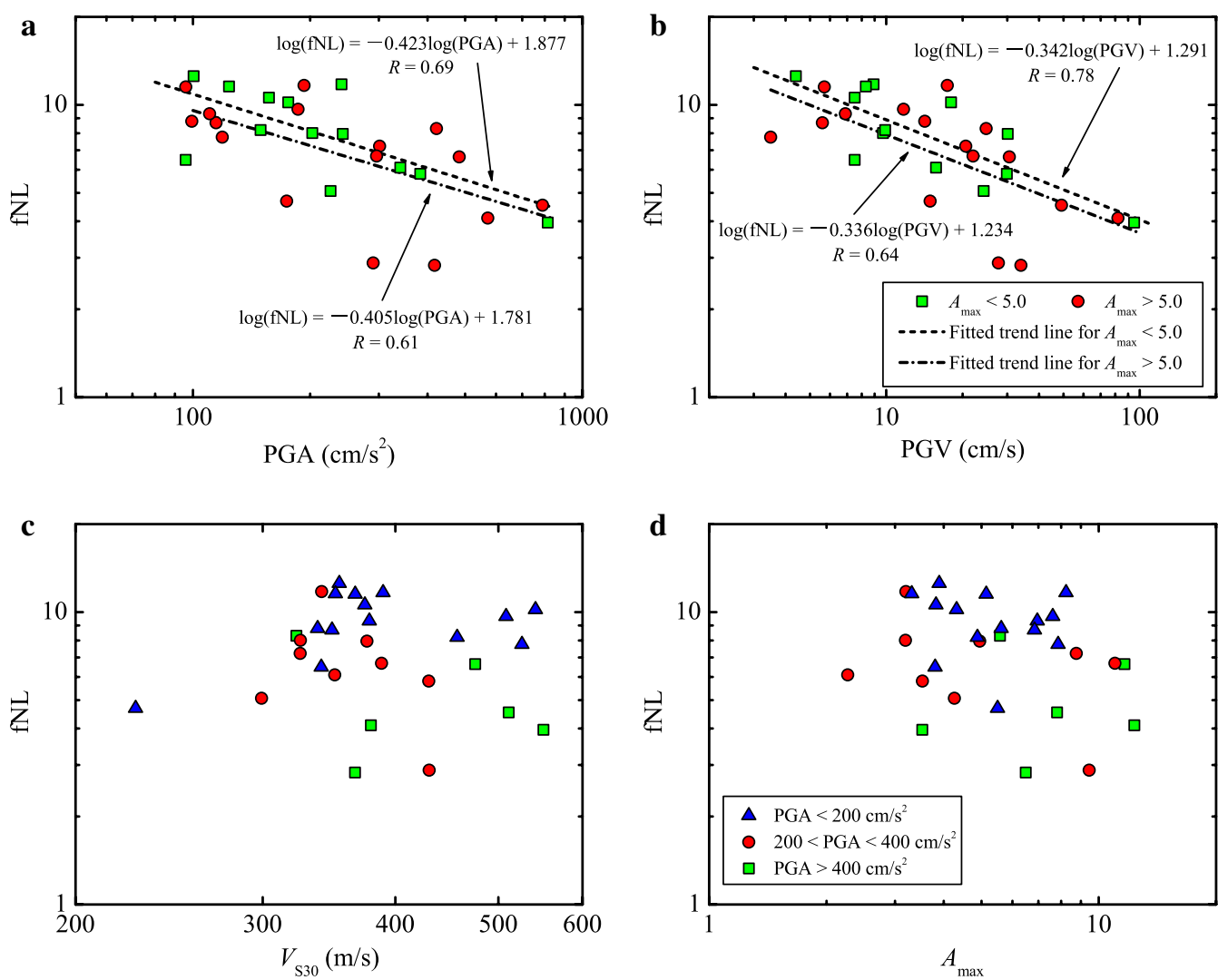

Fig. 10 The fNL values for 29 stations versus the recorded $\mathbf{a}$ PGAs and $\mathbf{b}$ PGVs during the Wenchuan Earthquake. The data were separated into two groups based on different $A_{\max }$ levels: $A_{\max }<5.0$ and $A_{\max }>5.0$. Linear fitting on a logarithmic scale was applied; the correlation coefficient $R$ is given for each regression. $\mathbf{c} f N L$ versus $V_{530}$ and $\mathbf{d}$ fNL versus $A_{\max }$. The data were separated into three groups based on different PGA levels: $P G A<200 \mathrm{~cm} / \mathrm{s}^{2}, 200<P G A<400 \mathrm{~cm} / \mathrm{s}^{2}$, and $P G A>400 \mathrm{~cm} / \mathrm{s}^{2}$

between fNL and $V_{\mathrm{S} 30}$ or $A_{\max }$, whichever group of data was analyzed. This is in accordance with the conclusion by Régnier et al. (2013) who determined a regression correlation coefficient of only 0.35 between $\mathrm{fNL}$ and $V_{\mathrm{S} 30}$. Therefore, the frequency width affected by soil nonlinearity predominantly depends on the ground-motion amplitude (e.g., PGA or PGV) rather than the site condition.

\section{Parameter PNL}

Figure 8c shows the correlations between PNL and PGA and PGV. The empirical relationships between these parameters were regressed using the following equation (Régnier et al. 2013):

$$
\mathrm{PNL}=a \cdot\{\tanh [\ln (\mathrm{PGA})-b]+1\},
$$

where $a$ and $b$ are the regression coefficients, that is, $a=23.77$ and $b=6.20$ for PGA and $a=20.72$ and $b=3.54$ for PGV, obtained in this study. The correlation coefficient is 0.73 for both PGA and PGV, revealing that PNL has a strong positive correlation with PGA or PGV, similar to the other parameters ( $\mathrm{RF}_{\mathrm{p}}, \mathrm{DNL}$, and $\left.\mathrm{ADNL}\right)$.
The empirical relationship between PNL and $A_{\max }$ was also regressed (Fig. 9d), indicating that PNL has a moderate positive correlation with $A_{\max }$, similar to DNL and ADNL.

\section{Discussion}

Our analysis shows that all five parameters have moderate correlations with PGA or PGV, proving the dependence of the soil nonlinearity on the ground-motion amplitude. The larger the ground motion is, the stronger the soil nonlinearity is. In addition, the soil nonlinearity also depends on the site condition; Fig. 9 shows that DNL, ADNL, and PNL positively correlate with $A_{\max }$. A larger $A_{\max }$ value generally corresponds to a stronger impedance contrast between the surface soil layers and overburden bedrock; therefore, the softer the soil layer is, the stronger the soil nonlinearity is. Note that stations 51MXT and 62WIX are located at rock sites, leading to small values of DNL, ADNL, and PNL, as shown in Fig. 8. Although the PGAs reached to 304.4 and $141.9 \mathrm{~cm} / \mathrm{s}^{2}$, respectively, there is no evidence of soil nonlinearity at both stations. 
The distribution of $\mathrm{RF}_{\mathrm{p}}$ versus PGA shown in Fig. 6 represents a PGA threshold of $200 \mathrm{~cm} / \mathrm{s}^{2}$ beyond which most stations evidently show nonlinear site response. The same PGA threshold was also derived from the regressed relationships between DNL and PGA shown in Fig. 8a based on a DNL threshold of 4.0 suggested by Noguchi and Sasatani (2011). Based on Fig. 4, the site responses under strong and weak motions do not differ much for most stations at which the recorded PGAs are almost below $200 \mathrm{~cm} / \mathrm{s}^{2}$ during the Wenchuan main shock.

Corresponding to the threshold of PGA (i.e., $200 \mathrm{~cm} /$ $\mathrm{s}^{2}$ ), the threshold of ADNL was proposed to be 0.2. Beyond this value, 10 stations exhibited evident soil nonlinearity. Two sites (51JZW and 51LXT) that were included when using the DNL parameter (see Fig. 8a, b) were excluded because the standard deviations are considered when using ADNL, making the difference between the HVSRs for S-waves during strong and weak motions smaller. When the standard deviation is large as calculating the average HVSR, it seems to be more scientific and reasonable to use ADNL rather than DNL.

The proposed threshold of PNL was 7\%, regarding a PGA threshold of $200 \mathrm{~cm} / \mathrm{s}^{2}$. Beyond this value, $13 \mathrm{sta}-$ tions exhibited evident soil nonlinearity. Most of them were also identified based on DNL and ADNL thresholds, except for 51JZB, 51MXN, and 51TQL. Both DNL and ADNL represent an absolute change of amplitude from linear to nonlinear site response, but PNL represents a relative change percentage. The amplitudes of the linear site response of stations $51 \mathrm{JZB}, 51 \mathrm{MXN}$, and $51 \mathrm{TQL}$ are not large (Figs. 3,4 ); in other words, the $A_{\max }$ values are small, that is, 3.90, 3.53, and 7.86, respectively. This could inherently increase the relative change from linear to nonlinear site response. Therefore, in this case study, the performance of PNL is better than that of DNL and ADNL.

Based on $\mathrm{RF}_{\mathrm{p}}$, DNL, ADNL, and PNL, 11, 12, 10, and 13 stations, respectively, were identified as sites with evident soil nonlinearity during the Wenchuan main shock (Table 2). The results of this identification might vary based on different parameters because each parameter has an individual feature; however, to identify sites with very strong soil nonlinearity (e.g., 51WCW or 51SFB), any one of above four parameters would be effective. It is not easy to conclude whether the performance of $\mathrm{RF}_{\mathrm{p}}$ is better or worse than that of DNL, ADNL, and PNL. For example, nonlinear site response was identified at station 51XJD using $\mathrm{RF}_{\mathrm{p}}$ but cannot be identified based on DNL, ADNL, and PNL (Table 2). For station 62WUD, the identification is ineffective using $R_{p}$ but effective using DNL, $A D N L$, and PNL. This is because $R_{p}$ focuses on the shift of the predominant frequency, but the three other parameters focus on the change of amplitude from linear to nonlinear site response. Figure 10 shows that parameter $\mathrm{fNL}$ is effective in evaluating the frequency band affected by soil nonlinearity, but less effective in identifying evidence of nonlinearity because this parameter cannot reflect the change of the predominant frequency or amplitude of the site response during weak and strong motions.

Table 2 Strong-motion stations with evident nonlinear site response during the Wenchuan main shock based on identification with $\mathrm{RF}_{\mathrm{p}}$, DNL, ADNL, and PNL, respectively

\begin{tabular}{|c|c|c|c|c|c|c|}
\hline \multirow[t]{2}{*}{ No. } & \multirow[t]{2}{*}{ Station code } & \multicolumn{4}{|c|}{ Parameters used to identify the soil nonlinearity } & \multirow[t]{2}{*}{ Nonlinearity level } \\
\hline & & $\mathrm{RF}_{\mathrm{p}}$ & DNL & ADNL & PNL & \\
\hline 1 & $51 \mathrm{AXT}$ & $\bullet$ & $\bullet$ & ○ & O & Medium \\
\hline 2 & $51 \mathrm{GYS}$ & $\bullet$ & • & ○ & • & Strong \\
\hline 3 & $51 \mathrm{GYZ}$ & $\bullet$ & $\bullet$ & $\bullet$ & $\bullet$ & Strong \\
\hline 4 & 51JYC & $\bullet$ & ○ & ○ & $\bullet$ & Strong \\
\hline 5 & 51JYD & $\bullet$ & ○ & ○ & ○ & Strong \\
\hline 6 & $51 \mathrm{JYH}$ & - & 0 & 0 & 0 & Strong \\
\hline 7 & $51 \mathrm{JZB}$ & 0 & 0 & 0 & 0 & Medium \\
\hline 8 & $51 \mathrm{JZW}$ & $\bullet$ & 0 & 0 & 0 & Medium \\
\hline 9 & $51 \mathrm{LXT}$ & 0 & 0 & 0 & 0 & Medium \\
\hline 10 & $51 M X N$ & 0 & 0 & 0 & 0 & Medium \\
\hline 11 & $51 \mathrm{MZQ}$ & $\bullet$ & 0 & 0 & 0 & Medium \\
\hline 12 & $51 \mathrm{SFB}$ & $\bullet$ & 0 & ○ & 0 & Strong \\
\hline 13 & $51 \mathrm{TQL}$ & 0 & 0 & 0 & 0 & medium \\
\hline 14 & 51WCW & 0 & 0 & 0 & 0 & Strong \\
\hline 15 & 51XJD & 0 & 0 & 0 & 0 & Medium \\
\hline 16 & 62WUD & 0 & • & - & 0 & Medium \\
\hline
\end{tabular}

and $O$ mean that the site response does and does not evidently behave nonlinearly 
Based on the proposed DNL, ADNL, and PNL thresholds of soil nonlinearity in conjunction with the fitted functions of DNL, ADNL, and PNL versus PGV, a PGV threshold of $\sim 15 \mathrm{~cm} / \mathrm{s}$ is proposed (Fig. 8). However, this threshold is $20 \mathrm{~cm} / \mathrm{s}$ based on the distribution of $\mathrm{RF}_{\mathrm{p}}$ versus PGV (Fig. 6). To maintain compatibility, we suggest $15 \mathrm{~cm} / \mathrm{s}$ as PGV threshold beyond which the site response potentially behaves nonlinearly.

As listed in Table 2, 16 stations were identified as sites with evident nonlinear site response during the Wenchuan main shock. According to the large values of $\mathrm{RF}_{\mathrm{p}}$, $\mathrm{DNL}, \mathrm{ADNL}$, and PNL, strong nonlinearity was suggested for seven stations, including 51GYS, 51GYZ, 51JYC, 51JYD, 51JYH, 51SFB, and 51WCW, and medium nonlinearity was suggested for the other nine stations. Evidence of soil nonlinearity can be observed at several other stations, such as 51CXQ, although not evident. Figure 4 shows that a slight reduction in the site response amplitude of the high-frequency band could be observed during strong motions. Therefore, we suggest a weak level of soil nonlinearity for such kind of stations. The level of soil nonlinearity for each station is presented in Table 1. Soil nonlinearity could not be observed at three stations (51MXT, 62SHW, and 62WIX) because 51MXT and 62WIX are located at rock sites and the PGA and PGV values (i.e., $99.7 \mathrm{~cm} / \mathrm{s}^{2}$ and $7.5 \mathrm{~cm} / \mathrm{s}$, respectively) at station $62 \mathrm{SHW}$ are small. Figure 11 shows the station locations with different colors of triangles indicating different soil nonlinearity levels. The contour map of PGA during the Wenchuan main shock is also shown in Fig. 11. The site responses of stations with a PGA $>200 \mathrm{~cm} / \mathrm{s}^{2}$ mostly behaved nonlinearly (strong and medium levels).

\section{Conclusions}

In this study, 33 strong-motion stations were selected to evaluate the soil nonlinearity during the 2008 Ms8.0 Wenchuan Earthquake using the HVSR method. Five parameters were calculated, $\mathrm{RF}_{\mathrm{p}}, \mathrm{DNL}, \mathrm{ADNL}, \mathrm{fNL}$, and

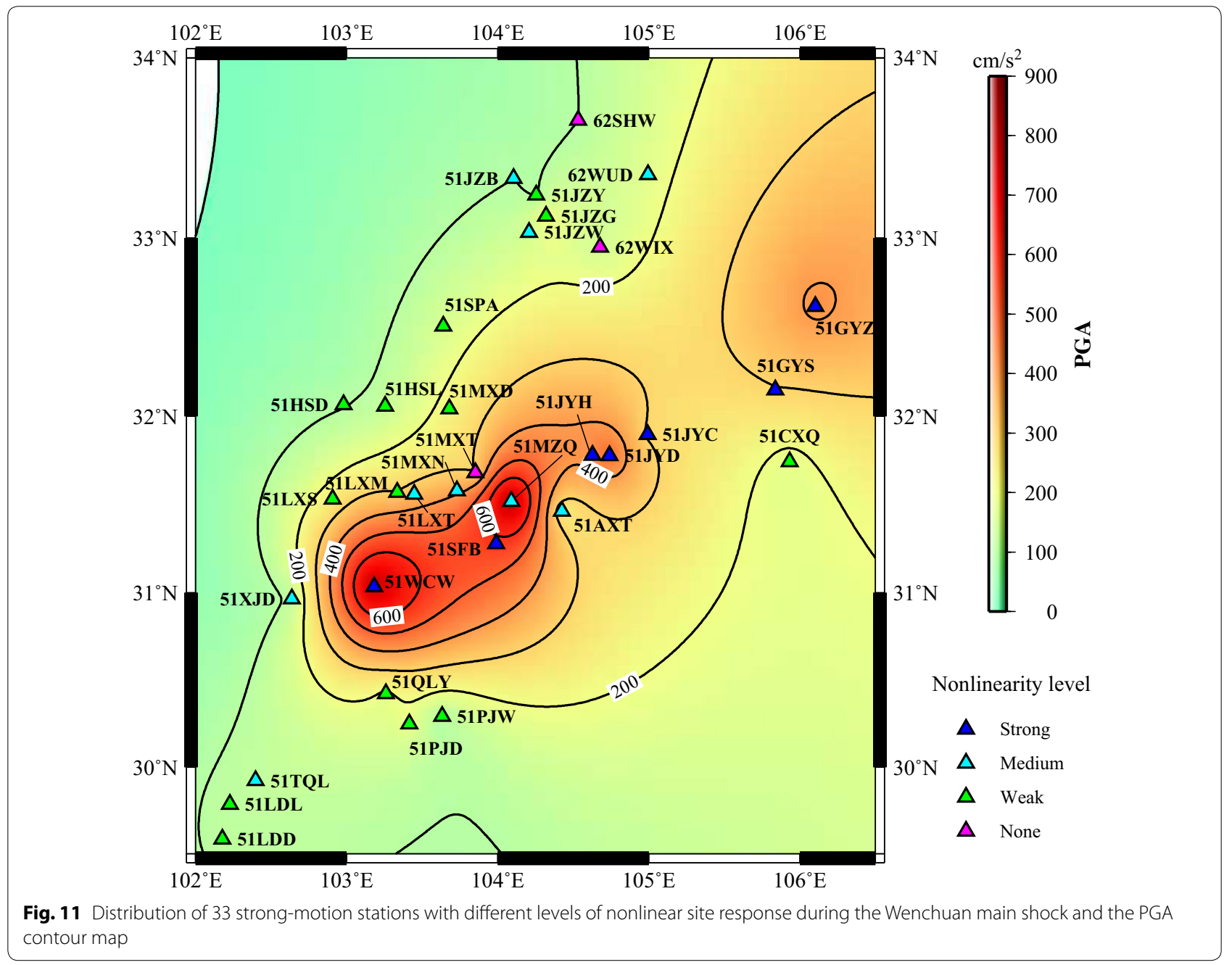


PNL, to characterize the nonlinear behavior of soil. The characteristics, performance, and effective usage of these parameters were analyzed, and their correlations with PGA, PGV, $V_{\mathrm{S} 30}$, and $A_{\max }$ were investigated. Based on this, the following conclusions were drawn:

(1) $\mathrm{RF}_{\mathrm{p}}, \mathrm{DNL}, \mathrm{ADNL}$, and PNL all have strong positive correlations with PGA and PGV, whereas fNL has a negative correlation. The DNL also correlates well with $A_{\max }$, but $\mathrm{fNL}$ shows no correlation with either $A_{\max }$ or $V_{\mathrm{S} 30}$. The empirical relationships between DNL and PGA and PGV, DNL and $A_{\max }$, $\mathrm{ADNL}$ and PGA and PGV, ADNL and $A_{\max }$, fNL and PGA and PGV, PNL and PGA and PGV, and PNL and $A_{\max }$ were all regressed, and moderate correlation coefficients were determined.

(2) Overall, 16 sites were found to exhibit strong and medium soil nonlinearity during the main shock of the Wenchuan Earthquake, where the groundmotion level was almost beyond a threshold of PGA $>200 \mathrm{~cm} / \mathrm{s}^{2}$ or PGV $>15 \mathrm{~cm} / \mathrm{s} ; 14$ sites exhibited weak nonlinearity; and 3 sites exhibited no nonlinearity. The thresholds of ADNL and PNL are proposed to be 2.0 and $7 \%$, respectively, beyond which the site response represents evident nonlinear behavior. Eight sites (51MZQ, 51WCW, 51SFB, 51JYD, 51JYC, 51JYH, 51GYZ, and 51GYS) could be identified using any of the four parameters, $\mathrm{RF}_{\mathrm{p}}$, DNL, ADNL, and PNL, whereas the other eight sites (51AXT, 51JZW, 51XJD, 51LXT, 51MXN, 51TQL, 51JZB, and 62WUD) could be identified using individual parameters. This study confirms that $\mathrm{RF}_{\mathrm{p}}$, DNL, $\mathrm{ADNL}$, and PNL all are effective in identifying the soil nonlinearity and can be selected based on user preference. The performance of PNL is better than that of DNL and ADNL in this case study.

(3) The good correlations between DNL (ADNL, PNL) and PGA and PGV and between DNL (ADNL, PNL) and $A_{\max }$ imply that the degree of soil nonlinearity not only depends on the ground-motion amplitude (e.g., PGA or PGV) but also on the site condition. However, the frequency width affected by soil nonlinearity predominantly depends on the ground-motion level rather than the site condition, as inferred by the phenomenon that $\mathrm{fNL}$ has a good correlation with PGA but does not correlate with $V_{\mathrm{S} 30}$ or $A_{\max }$.

\footnotetext{
Abbreviations

$A_{\text {max: }}$ : maximum amplitude of HVSR calculated using weak motions; ADNL: absolute degree of nonlinearity; DNL: degree of nonlinearity; $F_{\mathrm{p}}$ : site-predominant frequency; $\mathrm{fNL}$ : frequency of nonlinearity; GIT: generalized inversion technique; HVSR: horizontal-to-vertical spectral ratio; KiK-net: Kiban Kyoshin
}

network; K-NET: Kyoshin network; NGA: next-generation attenuation; PGA: peak ground acceleration; PGV: peak ground velocity; PNL: percentage of nonlinearity; $\mathrm{RF}_{\mathrm{p}}$ : ratio of predominant frequency; $V_{S 30}$ : average uppermost 30-m shear-wave velocity.

\section{Authors' contributions}

YFR analyzed the data, interpreted the results, and drafted the manuscript. RZW designed the study, interpreted the results, and made conclusions. XXY and $\mathrm{KJ}$ collected strong-motion records and processed the data. All authors read and approved the final manuscript.

\section{Acknowledgements}

The authors thank Kuo-liang Wen from the National Central University, Taiwan, for suggestions with respect to this study. We thank two anonymous reviewers for their valuable suggestions and constructive comments, which have considerably improved the quality of the manuscript.

\section{Competing interests}

The authors declare that they have no competing interests.

\section{Data and resources}

Strong-motion records used in this article were obtained from the China Strong-Motion Networks Center at http://www.csmnc.net/ (last accessed December 2012). The $V_{S 30}$ measurements were taken from the Next Generation Attenuation (NGA) site database of the Pacific Earthquake Engineering Research (PEER) Center at http://peer.berkeley.edu/ngawest2/databases/ (last accessed February 2015). Some of the figures were produced using Generic Mapping Tools (GMT).

\section{Funding}

This work was supported by the Science Foundation of the Institute of Engineering Mechanics, China Earthquake Administration (Grant No. 2016A04), National Natural Science Fund (No. U1534202), and Nonprofit Industry Research Project of the China Earthquake Administration (Grant No. 201508005).

\section{Publisher's Note}

Springer Nature remains neutral with regard to jurisdictional claims in published maps and institutional affiliations.

Received: 27 February 2017 Accepted: 8 August 2017

Published online: 22 August 2017

\section{References}

Aguirre J, Irikura K (1997) Nonlinearity, liquefaction, and velocity variation of soft soil layers in Port Island, Kobe, during the Hyogo-ken Nanbu earthquake. Bull Seismol Soc Am 87:1244-1258

Ancheta TD, Darragh RB, Stewart JP, Seyhan E, Silva WJ, Chiou BSJ, Wooddell KE, Graves RW, Kottke AR, Boore DB, Kishida T, Donahue JL (2013) PEER NGA-West2 database. PEER report, 2013/03

Beresnev IA, Wen KL (1996) Nonlinear soil response-a reality. Bull Seismol Soc Am 86:1964-1978

Beresnev IA, Field EH, Abeele KVD, Johnson PA (1998) Magnitude of nonlinear sediment response in Los Angeles basin during the 1994 Northridge, California, Earthquake. Bull Seismol Soc Am 88:1079-1084

Bonilla LF, Tsuda K, Pulido N, Régnier J, Laurendeau A (2011) Nonlinear site response evidence of K-NET and KiK-net records from the 2011 off the Pacific coast of Tohoku Earthquake. Earth Planets Space 63:785-789. doi:10.5047/eps.2011.06.012

Boore DM (2010) TSPP-A collection of FORTRAN programs for processing and manipulating time series. U.S. Geol. Surv. Open-File Rept. 2008-1111, revision 2.11

Borcherdt RD, Glassmoyer G (1992) On the characteristics of local geology and their influence on ground motions generated by the Loma Prieta earthquake in the San Francisco Bay region, California. Bull Seismol Soc Am 82:603-641

Celebi M, Prince J, Dietel C, Onate M, Chavez G (1987) The culprit in Mexico City-amplification of motions. Earthq Spectra 3:315-328 
Delépine N, Lenti L, Bonnet G, Semblat JF (2009) Nonlinear viscoelastic wave propagation: an extension of nearly constant attenuation (NCQ) models. J Eng Mech 135:1305-1314. doi:10.1061/ (ASCE)0733-9399(2009)135:11(1305)

Dhakal YP, Aoi S, Kunugi T, Suzuki W, Kimura T (2017) Assessment of nonlinear site response at ocean bottom seismograph sites based on S-wave horizontal-to-vertical spectral ratios: a study at the Sagami Bay area K-NET sites in Japan. Earth Planets Space 69:29. doi:10.1186/ s40623-017-0615-5

Dimitriu P (2002) The HVSR technique reveals pervasive nonlinear sediment response during the 1994 Northridge earthquake $\left(M_{w}\right.$ 6.7). J Seismol 6:247-255

Field EH, Johnson PA, Beresnev IA, Zeng Y (1997) Nonlinear ground-motion amplification by sediments during the 1994 Northridge earthquake. Nature 390:599-602

Hartzell S (1998) Variability in nonlinear sediment response during the 1994 Northridge, California, Earthquake. Bull Seismol Soc Am 88:1426-1437

Li XJ (2009) Uncorrected acceleration records from fixed observation for Wenchuan Ms8.0 aftershocks. Seismological Publishing House, Beijing (in Chinese)

Li XJ, Zhou ZH, Yu HY, Wen RZ, Lu DW, Huang M, Zhou YN, Cu JW (2008) Strong motion observations and recordings from the great Wenchuan earthquake. Earthq Eng Eng Vib 7:235-246

Noguchi S, Sasatani T (2008) Quantification of degree of nonlinear site response. In: 14th world conference on earthquake engineering, Beijing paper ID: 03-03-0049

Noguchi S, Sasatani T (2011) Nonlinear soil response and its effects on strong ground motions during the 2003 Miyagi-Oki intraslab earthquake. Zisin 63:165-187 (in Japanese with English abstract)

Pavlenko O, Irikura K (2002) Nonlinearity in the response of soils in the 1995 Kobe earthquake in vertical components of records. Soil Dyn Earthq Eng 22:967-975
Pavlenko O, Loh CH (2005) Nonlinear identification of the soil response at Dahan downhole array site during the 1999 Chi-Chi earthquake. Soil Dyn Earthq Eng 25:241-250. doi:10.1016/j.soildyn.2004.08.004

Pavlenko O, Wen KL (2008) Estimation of nonlinear soil behavior during the 1999 Chi-Chi, Taiwan, Earthquake. Pure Appl Geophys 165:373-407. doi:10.1007/s00024-008-0309-9

Régnier J, Cadet H, Bonilla LF, Bertrand E, Semblat JF (2013) Assessing nonlinear behavior of soils in seismic site response: statistical analysis on KiK-net strong-motion data. Bull Seismol Soc Am 103:1750-1770. doi:10.1785/0120120240

Régnier J, Cadet H, Bonilla LF, Bard PY (2016) Empirical quantification of the impact of nonlinear soil behavior on site response. Bull Seismol Soc Am 106:1710-1719. doi:10.1785/0120150199

Ren YF, Wen RZ, Yamanaka H, Kashima T (2013) Site effects by generalized inversion technique using strong motion recordings of the 2008 Wenchuan earthquake. Earthq Eng Eng Vib 12:165-184. doi:10.1007/ s11803-013-0160-6

Rong MS, Wang ZM, Woolery EW, Lyu YJ, Li XJ, Li SY (2016) Nonlinear site response from the strong ground-motion recordings in western China. Soil Dyn Earthq Eng 82:99-110. doi:10.1016/j.soildyn.2015.12.001

Trifunac MD, Todorovska MI (1996) Nonlinear soil response-1994 Northridge, California, Earthquake. J Geotech Eng ASCE 122:725-735

Wen KL (1994) Non-linear soil response in ground motions. Earthq Eng Struct Dyn 23:599-608

Wen KL, Beresnev IA, Yeh YT (1994) Nonlinear soil amplification inferred from downhole strong seismic motion data. Geophys Res Lett 21:2625-2628

Wen KL, Huang JY, Chen CT, Cheng YW (2011) Nonlinear site response of the 2010 Darfield, New Zealand earthquake sequence. In: 4th IASPEI/IAEE international symposium, 23-26 Aug 2011, Santa Barbara

Xu XR (2010) Recognition soil nonlinearity area applying the time-frequency analysis method in Wenchuan earthquake. Dissertation, National Central University, Taiwan

\section{Submit your manuscript to a SpringerOpen ${ }^{\odot}$ journal and benefit from:}

- Convenient online submission

- Rigorous peer review

- Open access: articles freely available online

- High visibility within the field

- Retaining the copyright to your article

Submit your next manuscript at $\boldsymbol{\nabla}$ springeropen.com 